\title{
Short-term variations in Class I methanol masers?
}

\author{
Preethi Pratap ${ }^{1}$, Vladimir Strelnitski ${ }^{2}$, Samantha Hoffman ${ }^{1}$ \\ and Jenna Lemonias ${ }^{2}$ \\ ${ }^{1}$ MIT Haystack Observatory, Westford, MA 01886 \\ email: ppratap@haystack.mit.edu \\ ${ }^{2}$ Maria Mitchell Observatory, Nantucket, MA \\ email: vladimir@mmo.org
}

\begin{abstract}
Class I methanol masers are found near regions of high-mass star formation and are pumped by collisions. Some indication of long-term variability in these masers has previously been reported (Kurtz et al. 2004). Here we will present evidence for variations in the intensity of the Class I methanol masers at $44 \mathrm{GHz}$ on much shorter timescales. We present preliminary results of the observation of the $44 \mathrm{GHz}$ Class I methanol transition toward sources in the DR21 region that indicates variability on timescales of days to possibly hours.
\end{abstract}

Keywords. masers, stars:formation

\section{Introduction}

Class I methanol masers are found around young, high mass star forming regions. They are most often associated with outflows and are often found offset from the star forming site and sometimes in regions with no obvious signs of star formation. In most of these isolated cases, signs of star formation are eventually detected. The masers are thus thought to probe a very early stage in the star formation process.

Methanol masers, until recently, have not been the subject of variability studies. Recent observations of the strong $6.7 \mathrm{GHz}$ Class II maser by Goedhart et al. (2004) found that these masers vary significantly with timescales on the order of days to years. Several sources were also found to have periodic variability. Different variability timescales need differing theories to explain the observations, such as small scale turbulence, shock activity resulting from outflow, intrinsic variation in the exciting sources and also possibly interstellar scintillation. In the case of Class I masers, long term variability has been reported in a few sources (Kurtz et al. 2004; Kalenskii et al. 2005) but no systematic measurements have been made. Systematic studies of the lower frequency transitions would provide added information regarding the nature and environment of these masers.

\section{Observations and results}

Data were taken with the Haystack 37-m telescope over several observing periods 2006 January, 2006 October and 2006 December. The observations in January were part of a mapping project and were not optimized to study the variability. However, over a period of a week there were indications of variability in DR21N, a newly discovered Class I methanol source, and DR21OH. The October 2006 observations were conducted more systematically, but some issues with pointing reduced the amount of usable data. Both the January and October data showed evidence of variability of the order of $>20 \%$ 
on timescales of days. The variability was determined from the ratios of strong maser components since this minimizes systematic errors.

The ratios of the intensities of two maser features in DR21OH were examined as a function of time over a period of several days in December 2006. The sources were observed from rise to set, except for about 3 hours at transit. The results show that the ratios change by about $20 \%$ in a period of $\tilde{6}$ hours. These particular maser features are situated within about 10 arcseconds of each other (Kurtz et al. 2004). This is important when studying the variation since the distribution of Class I masers in DR21OH is over an area of about $30^{\prime \prime}$. Variability detected in the ratios of other maser features that are separated by larger fractions of the Haystack antenna main beam ( $45^{\prime \prime}$ at $44 \mathrm{GHz}$ ) can be mimicked by pointing drifts.

\section{Discussion}

Possible short term variability in Class I methanol masers can put constraints on the excitation mechanism for these masers and on the source of the variability. One can rule out variability in the central radiative pumping source since the different velocity components vary differently. The variability could possibly arise in the small-scale turbulent medium in which the masers exist. Methanol masers appear to co-exist with $\mathrm{H}_{2} \mathrm{O}$ masers which also exhibit variability with timescales of 10's of days (Felli et al. 2006 and references therein). Some linear polarization that is detected in our data has also been seen in DR21-W at $133 \mathrm{GHz}$ (Wiesemeyer et al. 2004). Variability with a timescale $t$ can be used to set an upper limit on the size of the emitting area: $D<c t a^{1 / 2}$, where $a$ is the amplitude of the variation. Variations on timescales of a few hours results in an upper limit on the size of about $2 \times 10^{15} \mathrm{~cm}$, which is somewhat compatible with measured characteristic sizes of hot spots (Kogan and Slysh 1998). High angular resolution observations are needed to confirm the size limits.

The Class I methanol maser variability can be used to set constraints on the modeling of the maser excitation and the maser environment. These masers appear to be associated with a very early stage of star formation - one in which no near-IR or radio continuum sources are yet detected. Follow up, high resolution observations of the masers, the molecular gas and the continuum sources in the maser vicinity are needed to understand the mechanism for the variability. Observations of other sources are also needed to examine whether the variability is ubiquitous.

\section{Acknowledgements}

Support for undergraduate research with the 37-m telescope at MIT Haystack Observatory comes from the National Science Foundation (NSF). The REU programs at the Maria Mitchell Observatory and MIT Haystack Observatory are supported by grants from the NSF.

\section{References}

Felli, M., Massi, F., Robberto, M., \& Cesaroni, R. A\&A 453, 911

Goedhart, S., Gaylard, M. J., \& van der Walt, D. J. M.N.R.A.S, 355, 553

Kalenskii, S. V., Promyslov, V. G., Slysh, V. I., Bergman, P. \& Winnberg, A. 2005, Astronomy Reports 50, 289

Kogan, L. \& Slysh, V. 1998, Ap.J. 497, 800

Kurtz, S., Hofner, P. \& Alvarez, C. V. 2004, Ap.J.S. 155, 149

Wiesemeyer, H., Thum, C., \& Walmsley, C. M. A $\& A 428,479$ 


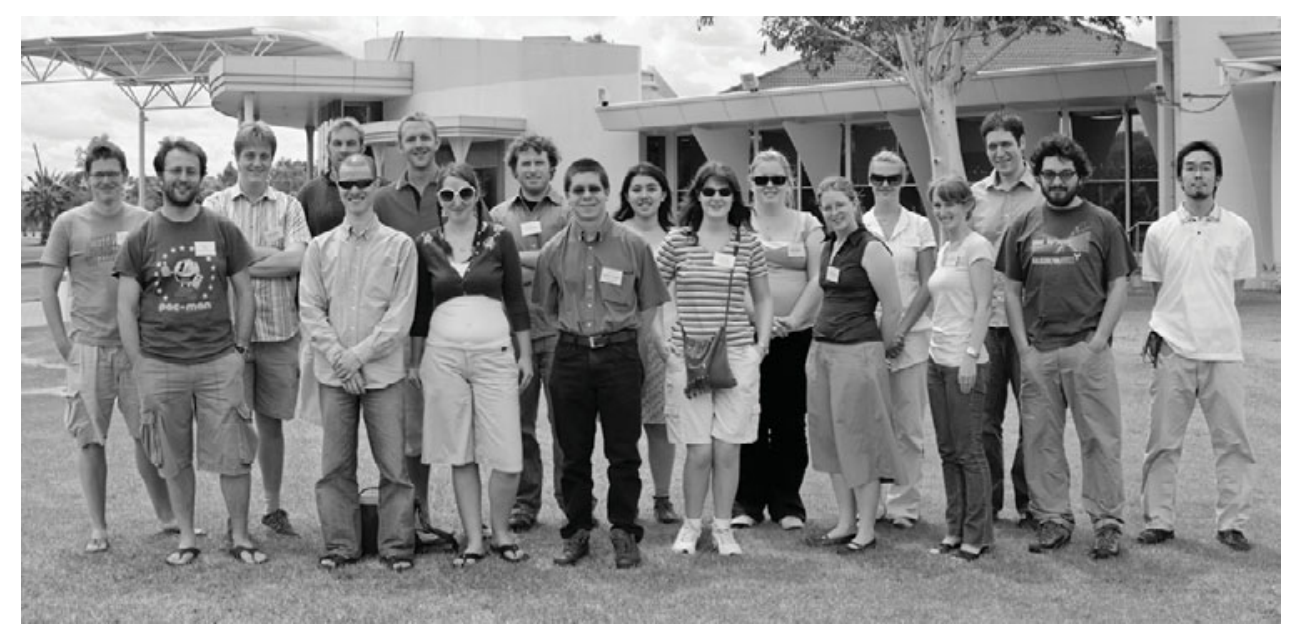

Photo: Shaun Amy

\section{PhD students at IAU 242}

From left to right: James Green, Carlos Gonzalez-Fernadez, Edo Loenen, Steven Longmore, Daniel Wong-McSweeney, Kalle Torstensson, Lyshia Quinn, Tim Robishaw, Estaban Araya, Mayumi Sato, Korinne McDonnell, Shari Breen,

Laura Richter, Alet de Witt, Kim McAlpine, Jack Hewitt, Rodrigo Parra, Koichiro Sugiyama (not shown: Daniel Tafoya, Paola Castangia) 